\title{
Uso de la Realidad Aumentada para fomentar la lectura
}

\section{Using Augmented Reality to encourage reading}

\section{Daniel Salas Álvarez¹, Diego Herrera Ferrer ${ }^{1}$, Eliecer Pérez Bohorquez ${ }^{1}$}

${ }^{1}$ Grupo Sócrates, Universidad de Córdoba, (Cra 6 No 77-305) Montería, Colombia; danielsalas@correo.unicordoba.edu.co; dfherreraferrer68@correo.unicordoba.edu.co; eperezbohorquez48@correo.unicordoba.edu.co

Recibido: 15/ago/2019 - Revisado: 30/sep/2019

Aceptado: 30/oct/2019 - Publicado: 30/jun/2019

Resumen El propósito de este trabajo es mostrar potencialidades de la Realidad Aumentada como herramienta tecnológica, para motivar la lectura en jóvenes en las instituciones educativas, además, que sirva como estrategia para enfrentar las dificultades que hoy se tienen en el aula, donde se evidencia una apatía generalizada de niños y jóvenes en relación con la lectura; para ello, se ha considerado el desarrollo de una aplicación de realidad aumentada aplicada a la obra El Principito, que se ha utilizado en jóvenes evidenciando el interés y motivación por este tipo de herramientas como estrategia para apoyar los procesos de lectura.

Palabras clave Realidad Aumentada, lectura, motivación, interés. Introduction (Heading 1)

Abstract The purpose of this work is to show the potentialities of Augmented Reality as a technological tool, to motivate reading in young people in educational institutions, in addition, which serves as a strategy to face the difficulties that today have in the classroom, where an apathy is evident generalized of children and young people in relation to reading; for this, the development of an augmented reality application applied to the work the little prince has been considered, which has been used in young people showing the interest and motivation for this type of tools as a strategy to support the reading processes.

Keywords Augmented Reality, Reading, Motivation, Interest. Introduction (Heading 1) 


\section{Introducción}

Se ha evidenciado por diversos estudios que las tecnologías de la información son herramientas conceptuales y técnicas que apoyan los procesos formativos en el aula, hoy se experimenta el uso creciente de tecnologías emergentes que son utilizadas en el contexto educativo, entre ellas, la Robótica, Impresión 3D, Inteligencia Artificial, Realidad Aumentada, Realidad Virtual, entre otras; frente a estas nuevas tecnologías que están emergiendo es necesario desarrollar iniciativas que permitan resolver dificultades apremiantes, debido a que cada día tienen mayor impacto en el aula y se ha observado con preocupación la apatía de los niños y jóvenes por la lectura.

Los estudios realizados por el DANE [1] a 2018, muestran que en general el promedio de lectura de libros en Colombia es de 2,9 libros por personas al año, estas cifras si bien muestran una mejoría con respecto a los años anteriores, son preocupantes frente a los Ranking internacionales; según los estudios de Global English Editing [2], los países con mejor promedio de lectura en el mundo, están liderados por India, Tailandia, China, Filipinas, Republica Checa, entre otros.

A nivel internacional las pruebas Pisa [3], evidencian que los países que tienen mayor rendimiento en lectura son Singapur, Japón, Estonia, China y Finlandia; en general, los países Latino Americanos, como Brasil, México Colombia, Perú, Costa Rica y República, se encuentra como coleros, en el estudio publicado por Pisa.

En relación con las competencias lectoras que se incluyen en el área de lenguaje en las pruebas Saber para 3, 5 y 9 grado, según los informes de la Gobernación de Córdoba [4], este departamento está entre los puestos 19 y 20 , a nivel nacional, con referencia a un total de 32 departamentos. Estos indicadores representan unas cifras que generan alertas y preocupación en la educación.
Por otra parte, se puede afirmar que la poca lectura de libros en la población colombiana, genera consecuencias en relación con la comprensión lectora, es decir, entre menos lectura haya, desde luego aumentaran las dificultades en la comprensión de textos, sin mencionar la incidencia en la lectura desde la perspectiva crítica.

Dada la situación expuesta, es necesario emprender acciones para intenten resolver esta dificultad presente en la educación del departamento de Córdoba; una de ellas, se plantea desde la incorporación masiva de tecnologías emergentes como herramientas pedagógicas que apoyen el proceso para motivar a los estudiantes a la lectura permanente, con sentido crítico.

\section{Estado del Arte}

Las tecnologías de la información y las comunicaciones, han tenido un vertiginoso crecimiento y desarrollo en la última década impactando este hecho a la sociedad en todos los ámbitos, económico, político, social y desde luego en lo educativo. Hoy se encuentran tecnologías emergentes que se reseñan en los estudios recientes y en reportes técnicos [5][6], como potenciales para generar nuevos desarrollos en la sociedad futura, entre ellas, se destacan, la impresión 3D, la Robótica, la Realidad Aumentada, el Blockchain y la Inteligencia Artificial, entre otras.

En este trabajo se aborda la Realidad Aumentada especialmente enfocada en los beneficios $y$ potencialidades para favorecer los procesos formativos, en este sentido, una primera aproximación de la aplicación de esta tecnología en educación según el planteamiento Steve Chi-Yin Yuen[7] que considera puede ser útil para la creación de libros[8], también se destaca la importancia de esta tecnología, desde el punto de vista de los juegos[9], que pueden ayudar a los estudiantes en el proceso de aprendizaje, de igual manera consideran que puede ser utilizada desde el punto de vista del aprendizaje por descubrimiento[10], 
además, plantean que la realidad aumentada puede utilizarse en la educación a través del modelamiento de objetos y para el desarrollo de habilidades y el entrenamiento[11][12].

Tal como se planteó anteriormente, se evidencia la utilización amplia de la Realidad Aumentada, en el campo de la educación, pero frente a su uso y ventajas, ¿Cuáles han sido los beneficios o impactos que ha logrado en los procesos formativos este tipo de tecnologías?, para ello, en un estudio de revisión sistemática de literatura realizado por Jorge Bacca[13], se evidencia que en relación con el uso de la Realidad Aumentada en educación, ha estado enmarcada para apoyar procesos de motivación, se ha utilizado como ejercicio de exploración, en procesos de colaboración, también para incrementar procesos de innovación, facilitar la interacción y crear actitud positiva en los estudiantes, entre otras.

Ahora bien, desde la perspectiva de la Realidad Aumentada en los procesos de lectura, se destaca el trabajo de Mary Cruz Palomares [14], desde el contexto de la comunicación literaria, vista a través de los libros interactivos, donde esta tecnología permite mayor versatilidad para la presentación de los contenidos de manera didáctica, ofreciendo a los lectores la posibilidad de jugar, conceptualizar y educar.

De acuerdo con el estudio de Kun-Hung Cheng [15], sobre la lectura de libros de Realidad Aumentada, afirma que en general, los estudiantes percibieron menos carga cognitiva, una motivación más fuerte y actitudes más positivas hacia las experiencias, al leer un libro basado en este tipo de tecnología.

\section{Metodología}

Este trabajo de investigación se desarrolló en tres fases, en la primera se desarrolló la aplicación de Realidad Aumentada, siguiendo el conjunto de actividades asociadas con la especificación de requisitos, diseño, implementación y pruebas del software; la segunda fase consistió en aplicar a los jóvenes las funcionalidades del aplicativo de Realidad Aumentada sobre los conceptos de la obra El Principito; y la tercera fase estuvo relacionada con los resultados alcanzados a partir de la aplicación en el escenario de pruebas.

\section{Resultados}

\subsection{Arquitectura de la Aplicación de RA}

La estructura de módulos y componentes de la aplicación de realidad aumentada está conformada por los elementos que se muestran en la figura 1.

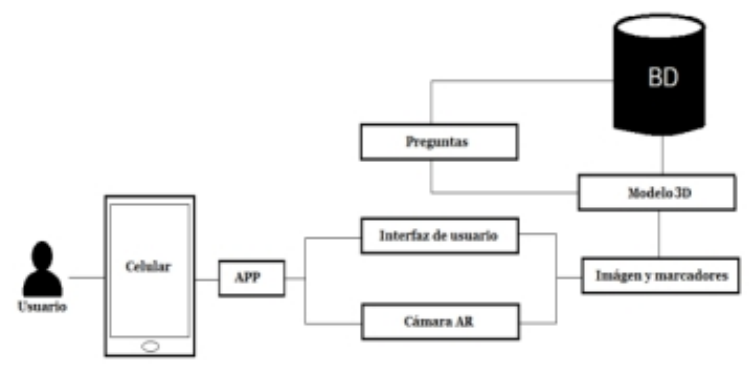

Fig 1. Arquitectura de la aplicación

Por otra parte, para el proceso de desarrollo, las herramientas que se utilizaron para crear la aplicación de El Principito fueron las Unity, Blender, Vuforia y Krita.

Unity es el motor de vídeo Juego que usamos para la programación en código C\#, Blender 3D para modelar los personajes, Krita para el texturizado de los modelos $3 \mathrm{~d}$ y vuforia es una herramienta para aplicar realidad aumentada con Unity.

\subsection{Aplicación RA basada en el principito}

Un esquema general de las funcionalidades de la aplicación, se presenta con el menú de opciones que contiene los iconos de las escenas que corresponden a la historia del libro El Principito, por lo que el usuario elegirá el icono para acceder a las escenas y al dialogo de la misma.

Luego de presentar las escenas en realidad aumentada al usuario, se realiza un breve cuestionario de la misma, que consta de cinco (5) preguntas que evalúan los aspectos esenciales mostrados en la aplicación, específicamente en cada escena; si el 
estudiante responde las preguntas de manera correcta, visualizará la siguiente escena.

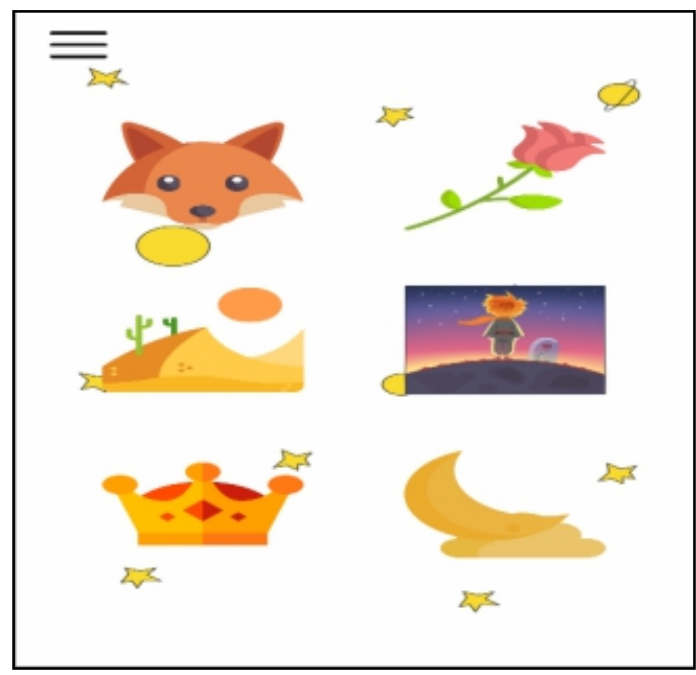

Fig 2. Esquema de interfaz de usuario.

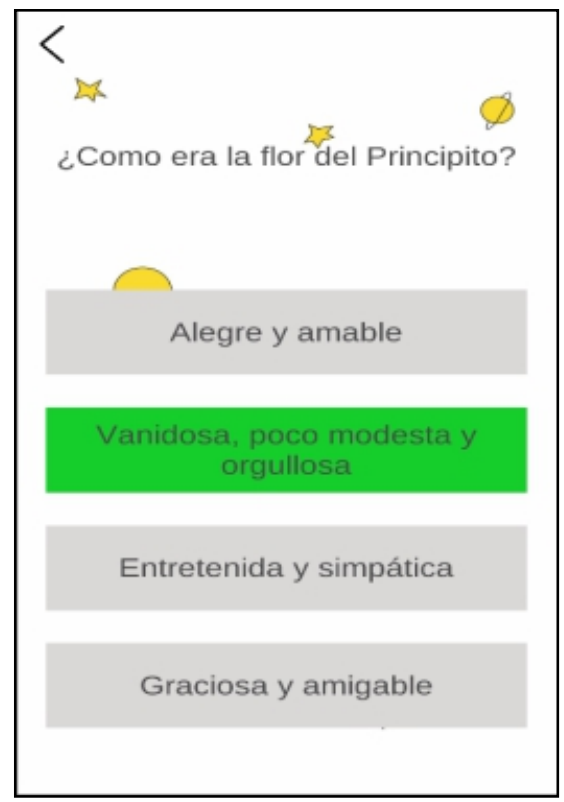

Fig 3. Presentación de evaluación

\subsection{Escenario de Prueba}

Se ha utilizado como escenario de prueba de la aplicación de Realidad Aumentada, al grado 8 de la Institución Educativa el INEM en la ciudad de Montería., La prueba fue realizada a 16 estudiantes con edades entre los 12 y 13 años, la cual contó con la participación de un docente de la Institución y el equipo que acompañó el proceso de apropiación del aplicativo, por espacio de una hora, donde se incluyeron las escenas de la obra El Principito, como estrategia para motivar a los estudiantes. Para ello, se les mostraron, todas las animaciones que conforman los principales planetas del libro, para que los jóvenes intentarán observar, analizar y representar esas conceptualizaciones y lograran tener sus propias interpretaciones que permitieran generar en ellos, la motivación para el leer el texto.

A continuación, se muestran tres escenas Fig.1, Fig. 2 y Fig. 3 del principito basados en tecnología de Realidad Aumentada que fueron presentadas a los 16 jóvenes de la Institución Educativa el INEM.

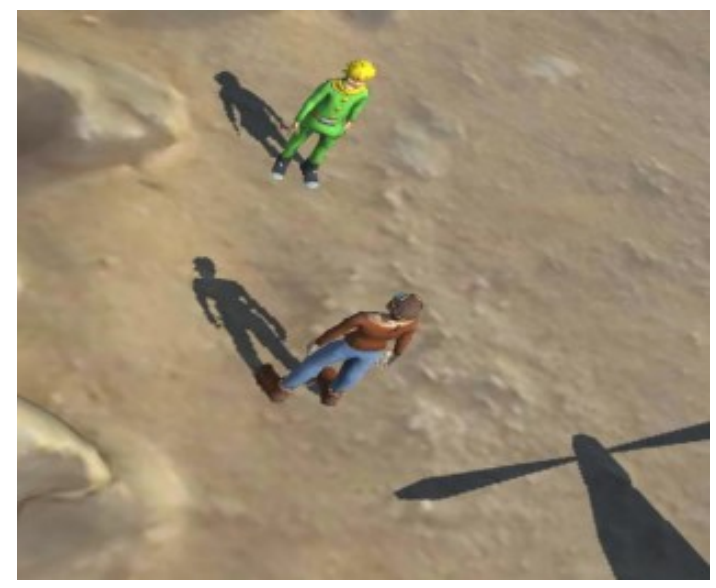

Fig 4. El principito y el piloto

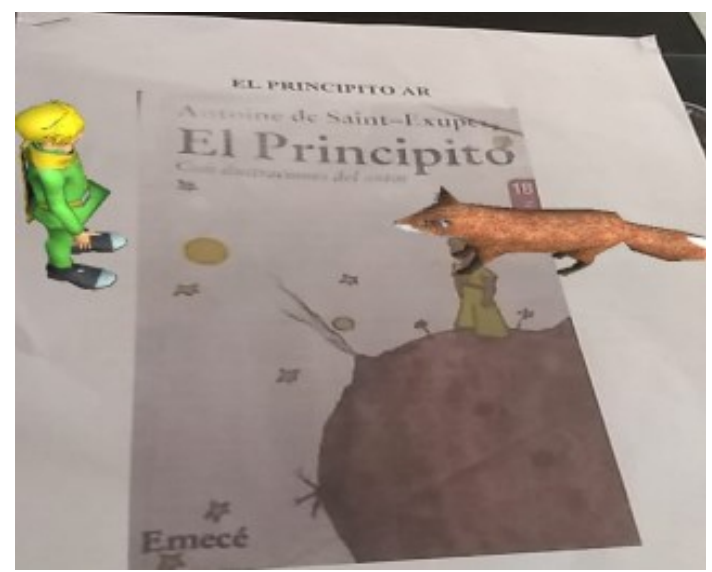

Fig. 5. El principito y el Zorro 


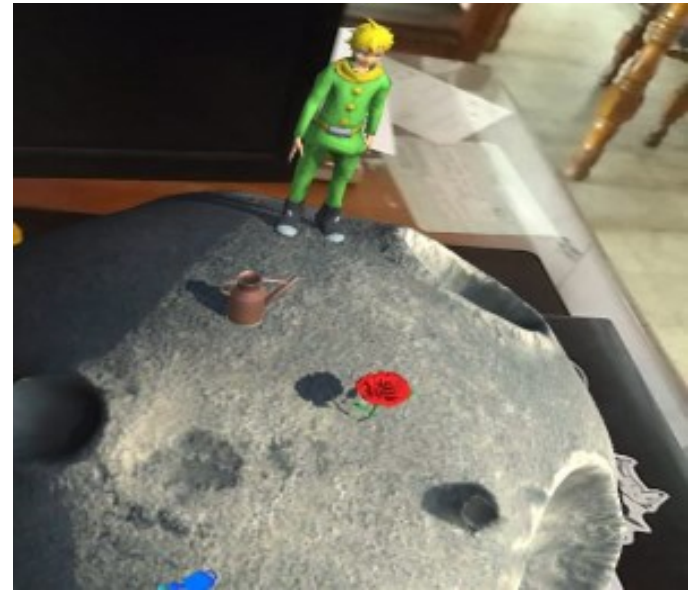

Fig. 6. El principito y la rosa

Los principales resultados de la aplicación de Realidad Aumentada a los 16 jóvenes de octavo grado de la Institución Educativa el INEM, se muestran en la tabla 1.

Tab 1. Resultados encuesta

\begin{tabular}{|c|c|c|}
\hline Pregunta & $\% \mathrm{Si}$ & $\%$ No \\
\hline $\begin{array}{l}\text { ¿Considera que le fue útil la } \\
\text { experiencia con el principito }\end{array}$ & 100 & 0 \\
\hline $\begin{array}{l}\text { ¿Se sintió atraído por la Aplicación el } \\
\text { principito? }\end{array}$ & 81,3 & 18,7 \\
\hline $\begin{array}{l}\text { ¿La experiencia con las actividades } \\
\text { de la aplicación, las consideró } \\
\text { interesante? }\end{array}$ & 86,3 & 13,7 \\
\hline $\begin{array}{l}\text { ¿Le interesaría seguir conociendo } \\
\text { más sobre el principito }\end{array}$ & 81,3 & 18,7 \\
\hline $\begin{array}{l}\text { ¿Le interesa conocer más sobre el } \\
\text { principito, por lo que representa su } \\
\text { filosofía de vida? }\end{array}$ & 60 & 40 \\
\hline $\begin{array}{l}\text { ¿Le gustaría que se mejoraran de la } \\
\text { Aplicación en animaciones y } \\
\text { actividades? }\end{array}$ & 70 & 30 \\
\hline ¿Qué tan fácil es utilizar la aplicación? & 100 & 0 \\
\hline ¿Recomendaría esta Aplicación & 100 & 0 \\
\hline Promedio Porcentaje & 84,6 & 15,4 \\
\hline
\end{tabular}

Los principales comentarios y aportes surgidos a partir del escenario de prueba, muestran las apreciaciones, aportes y sugerencias de los estudiantes en relación con la aplicación de Realidad Aumentada para fomentar la lectura.

\section{Comentarios:}

- Me gustó la aplicación por las animaciones.

- Me gustaría conocer más sobre El Principito es muy interesante la historia y divertida.

- Quiero conocer el libro con la aplicación para ver y saber cómo son los personajes.

- Me parece un libro muy bueno para aprender.

- Se pueden mejorar las animaciones de los personajes.

- Mejorar las preguntas de la evaluación para que no se repitan.

- Que realicen más animaciones.

- Cuando vaya a leer el libro con la aplicación. Me gustó mucho porque nos anima a leer.

- Me gustó mucho porque fue una experiencia 3D.

- Me gustaría que mejorarán los gráficos de los personajes.

\section{Conclusiones}

A partir de la implementación de la aplicación de Realidad Aumentada se puede concluir es una herramienta tecnológica que puede motivar y animar a los niños y jóvenes a la lectura, dada las facilidades y potencialidades para la interacción y participación de los mismos, igualmente se evidenció en la experiencia relacionada con el uso de la aplicación de El Principito, que hubo interés y motivación para seguir explorando este tipo de alternativas con el objetivo de que los estudiantes tenga mayores posibilidades de leer textos y puedan ser de ayuda para mejorar las competencias lectoras.

Trabajo Futuro La proyección de este trabajo está relacionada con dos aspectos, el primero es considerar un estudio experimental para observar detalladamente el comportamiento de las variables, por otra parte, realizar mejoras a la aplicación en cuanto a la interfaz, gestión de datos y desarrollo de nuevas animaciones.

\section{Referencias}

DANE, Encuesta de Consumo Cultural del Dane, 2018, Ministerio de la Cultura. 
Global English Editing, World Reading Habits in 2018 (Infographic).

Gobernación del Departamento de Córdoba, Boletin estadístico, Educación articulada para La Paz.

OCDE (2017), Marco de Evaluación y de Análisis de PISA para el Desarrollo, Lectura, matemáticas y ciencias, Versión preliminar, OECD, Publishing, Paris.

A.Becker, M. Brown, E. Dahlstrom, A. Davis, K. DePaul, V., Diaz, and J., Pomerantz. NMC Horizon R report: 2018 Higher Education Edition. Louisville, CO: EDUCAUSE, 2018.

A. Becker, S., Cummins, M., Davis, A., Freeman, A., Hall Giesinger, C., and Ananthanarayanan, V. (2017). NMC Horizon Report: 2017 Higher. Education Edition. Austin, Texas: The New Media Consortium.

S. Yuen, G. Yaoyuneyong, Augmented Reality: An Overview and FiveDirections for AR in Education, 2011

S. Yuen, S. -Y. (2010, November 19). 3D Augmented Reality Books. RetrievedJuly 21, 2011 from http://steveyuen.org/blog/?p=754

Dunleavy, M., C. Dede, \&R, Mitchell. Affordances and limitations of immersive participatory augmented reality simulations for teaching and learning. Journal of Science Education and Technology,18(1), 7-22, 2009

R., De Lorenzo, Augmented Reality and On-Demand Learning. The Mobile Learner.Retrieved July 22, 2010 from http://themobilelearner. wordpress.com/2009/10/17/ augmented-reality-andon-demand-learning/, 2009

S. Saenz,.Augmented Reality To Help Military Mechanics Fix Vehicles(Video). fromhttp://singularityhub.com/2010/01/11/augmented -reality-to-help-military-mechanics-ix-vehicles-video/, Retrieved July 20, 2011

Azuma, R. T. (1997, August). A survey of augmentedreality. In Presence: Teleperators and Virtual Environments, 6(4), 355-385.

J. Bacca, R. Fabregat, S. Baldiris, S Graf, Augmented Reality Trends in Education: A Systematic Review of Research and Applications.

M. Palomares.: La Realidad Aumentada en la comunicación literaria. El caso de los libros interactivos", en ENSAYOS, Revista de la Facultad de Educación de Albacete, № 29-2, 2014. (Enlaceweb: http://www.revista.uclm.es/index.php/ensayos
K, Chen, Reading an augmented reality book: an exploration of learners' cognitive load, motivation, and attitudes, Australasian Journal of educational technology, Vol 33, 4. 